\title{
SYPHILIS OF THE CENTRAL NERVOUS SYSTEM.
}

\author{
By B. BUCKLEY SHARP, M.D., M.R.C.P. \\ (Physician to the Princess Beatrice and Evelina Hospitals; \\ Assistant to the Neurological and Venereal Disease Depts., Royal Northern Hospital; \\ Assistant in the Venereal Disease Dept., St. Bartholomew's Hospital.)
}

\section{Clinical Features, Diagnosis and Pathology.}

Disease processes due to the treponema pallidum (or spirochæta pallida) are found most commonly in the vascular system, so that most of the lesions produced in the various organs of the body can be traced to disorder of the vascular supply. They are chronic inflammatory lesions passing through the stages of congestion, perivascular infiltration with small round cells and plasma cells, granulation tissue, and finally fibrous tissue. The granulation tissue may be sufficient in amount to form a tumour. The arterioles themselves become obstructed by endarteritis obliterans or the formation of thrombi on their diseased walls, and the areas supplied by them become infarcted and fibrotic. Myocardial degeneration occurs in this way, as may also degeneration of the walls of larger arteries supplied by vasa vasorum, paving the way to the development of an aneurysm. Syphilitic granulation tissue becomes necrotic with the formation of a gumma.

These mesodermal lesions arise as readily in the central nervous system as in any other part of the body and are termed cerebro-spinal, or meningo-vascular, syphilis. They may give rise to hæmorrhage and thrombosis, to a chronic form of meningitis or to gumma formation within the skull, and to the same conditions within the spinal canal. Within the cranial cavity a gumma behaves like a neoplasm, and may cause death from raised intra-cranial pressure. Syphilitic lepto-meningitis resulting first in infiltration of the membranes, then thickening and scarring, frequently produces cranial nerve palsies, the sixth and third nerve trunks being the most frequently involved with corresponding ophthalmoplegias, external only in the case of the sixth nerve (abducens paralysis), and both external and internal (large inactive pupil), in the case of the third nerve. Ophthalmoplegia from this cause must be diagnosed from the external ophthalmoplegias of toxic polyneuritis, Graves' disease, orbital periostitis, tumour or gumma of the orbit, central vascular lesions, primary nuclear degenerations, cerebral contusion, . pituitary tumour, cavernous sinus thrombosis, encephalitis lethargica, myasthenia gravis, raised intracranial pressure, and more rarely the brain-stem forms of poliomyelitis, syringomyelia and progressive muscular atrophy. The differential diagnosis of internal ophthalmoplegia will be discussed under the heading of tabes dorsalis.

Occasionally a sub-arachnoid block from syphilitic meningitis may cause hydrocephalus, the signs suggesting an unlocalisable tumour. Epileptiform fits are a common accompaniment of cerebral syphilis. Mention should also be made of syphilitic pachymeningitis, the cardinal symptom of which is headache, often unilateral, of a sharp stabbing character.

Within the spinal canal the same lesions occur, but, of course, the clinical manifestations are different owing to anatomical differences. Syphilitic spinal meningitis usually affects the cervical region of the cord, and the signs and symptoms may be unilateral. The initial symptom is pain in the back, between the shoulders and down one or both arms. After a considerable interval, there is paresis of the lower motor neurone type with muscular wasting and sensory loss corresponding to the roots involved; a tumour, a neuritis or a cervical rib may be 
suspected, syringomyelia being excluded by absence of dissociated sensory loss. If the meningitis extends down to the first dorsal segment, a Horner's syndrome will be found on the same side. A similar affection over the lumbar enlargement may be confused with "sciatic" pain from other causes.

When the substance of the cord itself is involved, the condition is one of myelitis, the clinical picture being due to vascular occlusion. Myelitis is commonly associated with a meningitis, and syphilitic meningo-myelitis may be acute or chronic. The dorsal region of the cord is the site of election. In the acute form a transverse lesion of the cord may develop in the course of a few days. In the chronic form the early symptoms are pain in the back and tenderness of the spine with radiating pain in the limbs or round the trunk. Cord symptoms appear after a shorter or longer interval with weakness of the legs progressing to spastic paraplegia, numbness and parasthesiæ. Sensory changes are not necessarily as severe as the motor symptoms. Temperature sense and vibration sense are often deranged. Bladder control is often impaired. There is a rare type in which sensory loss is minimal and signs of meningeal involvement absent, but with spastic weakness of the lower limbs, known as Erb's syphilitic spinal paralysis. A case of chronic syphilitic meningo-myelitis must be diagnosed from disseminated sclerosis, cord tumour, subacute combined degeneration, arachnoiditis and amyotrophic lateral sclerosis. In the latter condition, although there is no sensory loss, there may be aches, pains, cramps and parasthesiæ in the parts affected; sphincter disturbance is unusual. The characteristic muscular wasting with fibrillary tremor is rare in spinal syphilis, but has been occasionally described. Syphilis is thought to be a predisposing cause of progressive muscular atrophy in some cases.

The lesions so far mentioned do not occur till the syphilitic infection has been in existence in the body for some months or years. Acute syphilitic meningitis, may, however, occur in the secondary stage of the disease, and may coincide with the secondary rash.

Though meningo-vascular syphilitic lesions are the more common, parenchymatous involvement of the central nervous system occurs not infrequently, constituting what is sometimes termed the "parasyphilis" group. The tissues involved in this case are of ectodermal origin, and the onset is late. Parenchymatous and meningo-vascular lesions may co-exist.

Parasyphilis comprises :-

I. Chronic syphilitic encephalitis (dementia paralytica).

2. Tabes dorsalis.

3. Tabo-paresis.

Their onset is rarely earlier than 5 years after infection; the maximum incidence is about II years after infection; an interval of 30 years is not uncommon; the longest interval in the writer's own experience was 40 years.

Dementia paralytica, (G.P.I.) in its fully developed stage is characterised by mental changes of manic, grandiose, depressed, paranoid or simple dementing types. Typically there are Argyll Robertson pupils, dysarthria, tremor and exaggerated knee jerks. Epileptiform seizures, called congestive attacks, may occur, but are not confined to dementia paralytica, occurring also in cerebral syphilis. The appetite may be voracious. 
Superficially there may be points of resemblance between dementia paralytica and frontal lobe tumour, in which mental changes, often of euphoric type, and epileptiform seizures may also be observed. The presence of papilloedema would favour a diagnosis of tumour. The condition of alcoholic pseudo-paralysis, which must be extremely rare, is said to show sluggish pupils, tremors of the face (chiefly of the upper part), a rippling tremor of the tongue as distinguished from the "trombone" tremor of dementia paralytica, coarse tremor of the hands, absent knee jerks if there is neuritis, severe mental confusion with delusions, and tremulous articulation and writing.

It is a disaster if the diagnosis of dementia paralytica is delayed till the clinical picture described above has developed. It cannot be over-emphasized that minor mental and personality changes precede as a rule any demonstrable neurological signs. The early diagnosis is usually one of " neurasthenia " or of minor psychosis such as autonomous depression. The development of such changes de novo calls for serological examination in every instance even if there appears to be an adequate extraneous cause for a state of anxiety or depression. The chances of successful treatment depend in large measure on the duration of the disease before treatment is commenced. Similarly the investigation of no disorder of the nervous system is complete till evidence of syphilis has been sought.

Tabes is a neuro-syphilitic affection mainly involving the posterior roots and posterior columns of the cord, and the mid-brain. The retinal nerve cells may suffer damage with resulting optic atrophy and amaurosis. It remains to be explained, however, why central scotoma is so rare, the more common clinical type being that of initial loss of peripheral vision. In fact there is considerable doubt whether degeneration starting at the macula, and thus producing a central scotoma, has ever been satisfactorily proved to be the result of syphilis. To explain this phenomenon Kinnier Wilson postulated interstitial lesions round the periphery of the optic nerves as the lesion in tabetic optic atrophy. L. Paton describes two types of visual failure in tabes, a uniform diminution of visual acuity with no field defects in the early stages, and a type with retention of good central vision but with peripheral or other field defect. The first type he regards as parenchymatous degeneration of the retinal nerve cells and fibres; the second type he regards as due to interstitial lesions invading the nerve from the pial sheath. In the latter case the intra-cranial portions of the nerves are first involved. $H$. Lauber has recently stressed the importance in optic atrophy of the relationship between the diastolic pressure in the retinal arteries and the intra-ocular pressure, stating that when the former is less than $20 \mathrm{~mm}$. of mercury higher than the latter the capillary circulation is impeded and the nutrition of the retina impaired. Hypotonia is, according to Lauber, common not only in tabes, but also in Leber's hereditary optic atrophy, in anæmia from blood loss, and in retinitis pigmentosa. Optic atrophy, when present, is usually an early phenomenon. Those cases of tabes in which it occurs, though they may go on to blindness, become arrested in other respects and do not progress to marked ataxia.

In the earlier, or pre-ataxic, stage "lightning" pains may occur, often as the first symptom. Their character is typical-a sudden brief stabbing pain as if a hot needle had been run into the part. They are often felt in the neighbourhood of joints, the lower limbs being the usual site, though they may be felt round the trunk or, indeed, in any part of the surface of the body. They come on in bouts 
with periods of freedom. In considering the ætiology of these pains, it is of interest to consider other conditions in which similar pains arise:-

(a) Extra-medullary tumours in the spinal canal cause root pains at the affected segmental level. But, in addition, both extra- and intra-medullary tumours may cause pains referred to parts of the body below the affected segmental level, these pains being aching or boring in character rather than lancinating, persistent, and aggravated by movement, vibration and external stimuli.

(b) Syringo-myelia of the cervical cord is occasionally accompanied by pains in the legs of burning or cutting character or painful sensations of cold.

(c) Disseminated sclerosis in its early stage may occasionally be accompanied by spontaneous, intermittent, shock-like pains especially affecting the legs. Rarely there are girdle pains. Severe trigeminal neuralgia has been observed by Wilfred Harris to accompany disseminated sclerosis not infrequently.

(d) Subacute combined degeneration is accompanied by parasthesiæ; but, in addition, severe pains in the legs may be complained of.

(e) Vascular disease, especially in elderly hyperpietics, may give rise to shooting pains in the lower limbs.

The pains in the five conditions mentioned above (except the segmental root pains in extra-medullary tumour) are examples of central pain: i.e., peripheral pain due to lesions in the substance of the spinal cord.

There are still two other conditions in which pains in the lower limbs may be mistaken for tabetic lightning pains:-

1. Diabetes in which the pains are usually attributed to neuritis. But as the deep reflexes are often retained, and as the patients affected are mainly the elderly arteriosclerotic diabetics, a vascular origin is likely.

2. Arachnoiditis, which is a chronic condition exhibiting adhesions in the subarachnoid space, sometimes with local encystment of cerebro-spinal fluid usually without any definite cyst wall, and sometimes a more general matting without any local collection of fluid. The arachnoid is in intimate relationship to the posterior nerve roots forming a wrapping round them, its relationship with the anterior roots being much less intimate. Thus posterior root symptoms are the earliest phenomena in spinal arachnoiditis and take the form of peripheral pains, often intermittent and paroxysmal and not confined to one area. These pains may precede any sensory loss or objective neurological signs by several years. With the slow progress of the disease sensory loss, diminished reflexes, some involvement of the anterior roots with muscular wasting and fibrillary tremor occur, and finally compression of the cord. Sphincter involvement is late. Frequently microscopic examination fails to reveal inflammatory changes, and the cerebro-spinal fluid shows no pathognomonic changes unless there is a subarachnoid block. It would clearly be easy on clinical grounds to diagnose the pains of early arachnoiditis as tabetic lightning pains; a helpful distinguishing point is that arachnoiditis is often accompanied by pain over the spine itself, which does not occur in tabes. The two conditions may, however, co-exist.

What then is the site of the pain-producing lesion in tabes? Feiling has recently expressed the view that tabetic lightning pains may be due to central lesions affecting the intra-spinal course of the nerve fibres from the posterior root ganglia, in support of which he points out the local and non-segmental distribution, that they may be the first symptom, and that they may persist when no serological 
evidence of disease remains in the cerebro-spinal fluid or blood, their persistence being no indication, therefore, of progress of the disease. He holds that the theory of chronic meningitis causing pressure on the posterior roots at their entrance to the cord (Obersteiner \& Redlich) fails because of the absence of pathological and serological evidence of meningitis in many cases, and the absence of such pains in known meningitis; that the theories of radiculitis (Nageotte), and of specific granuloma of the radicular nerve (Richter), fail because of the absence of involvement of anterior roots and the inability of Stern (I929), to confirm Richter's findings.

It remains to mention the theory of Foerster (I927), who postulates a special nerve pathway from the cerebral cortex to the lateral column of the cord close to the posterior horn, which exerts an inhibitory effect upon the pain-subserving function of this horn. Destruction of this pathway would permit of the occurrence of pains as a release phenomenon (c.f. thalamic syndrome). This theory appears to be the only one supporting the central origin of tabetic pains that conforms to the usual conception of the transmission of pain sensation across to and up the antero-lateral column of the opposite side. It appears to the writer that in the light of a study of spinal arachnoiditis, it is difficult to reject altogether a meningeal ætiology of tabetic lightning pains.

The objective sensory changes in tabes consist of patchy hyperæsthesia, particularly to cold stimulation of the skin, and, as the disease progresses, sensory loss develops in the shapes of areas of analgesia, either patchy or of segmental distribution. It is common to find it under the balls of the toes, over the peroneal aspects of the legs, as a band on the trunk, on the tip of the nose, or as diminished sensation when the ulnar nerve is pressed at the elbow. The next stage of sensory loss involves joint and muscle sense and vibration sense. The centripetal fibres of the bipolar nerve cells in the posterior root ganglia that subserve these sensations have the longest course in the spinal cord, as they have no cell station till they reach the nuclei of Goll and Burdach in the medulla. It follows that the lower limbs are affected before the upper limbs. Breaking of the reflex arc on the afferent side results in loss of deep reflexes at the appropriate level and for muscular hypotonia; usually the ankle jerks are lost before the knee jerks.

Viscera supplied by the autonomic nervous system may be affected by the upsetting of the balance of sympathetic and parasympathetic action. Thus visceral crises may be found early in the disease, gastric crises being the most common and characterised by epigastric pain and vomiting with hypersecretion of acid gastric juice. Laryngeal crises, and rectal crises with left iliac fossa pain and constipation are far less common. Vesical crises in which there are attacks of frequent and painful micturition are also rare. When the disease is more advanced and there is interruption of the reflex arc on the afferent side, voluntary control of micturition is impaired, and finally painless retention occurs.

Ataxia in tabes is, of course, due to loss of sensory information from joints and muscles. The patient has to guide his movements and maintain his posture by ocular control and by voluntary instead of reflex muscular action. In the dark, or with the eyes closed, he cannot maintain his balance. This is the basis of Romberg's test. Cerebellar ataxia, being due to inco-ordination of movement and not to sensory loss, only reveals itself when the patient begins to move or walk, and Romberg's test is negative. In Friedreich's ataxia, as there may be degeneration of the posterior columns as well as of the cerebellum, the ataxia may show features of both types. 
The trophic effects of tabes occur in the later or degenerative stage, and are therefore not associated with any marked degree of pain, the most common being perforating ulcer of the foot and Charcot's joint. Their painlessness is in part their cause, as the part is thus not protected from trauma. A perforating ulcer will heal with rest in bed for a sufficient length of time. Fractures occur with abnormally slight injury, but re-unite under appropriate treatment. A tabetic Charcot's joint is usually either a knee or an ankle. The Charcot's joint of syringo-myelia is found in the upper limb. Perforating ulcer is also found in diabetes past middle age, where vascular degeneration becomes a factor.

The mid-brain effects commonly pick out the region of the aqueduct and the anterior corpora quadrigemina, causing loss of pupil reaction to light by interference with the afferent path from the retina to the nucleus of the third nerve. It is obvious that a lesion of the nucleus itself or of the efferent path therefrom would cause loss of pupil reaction to convergence as well. The afferent nerve fibres from the anterior corpora quadrigemina cross to the anterior end of the third nerve nucleus of the opposite side, and in doing so pass partly behind and partly in front of the aqueduct, and intermingle with the fibres of the tecto-spinal tract. If the tecto-spinal tract is damaged, contraction of the pupils (myosis) results from loss of the action of the cervical sympathetic. The reflex arc for dilatation of the pupil runs from the anterior corpus quadrigemens via the tectospinal tract to the first dorsal segment of the cord, whence the cervical sympathetic emanates.

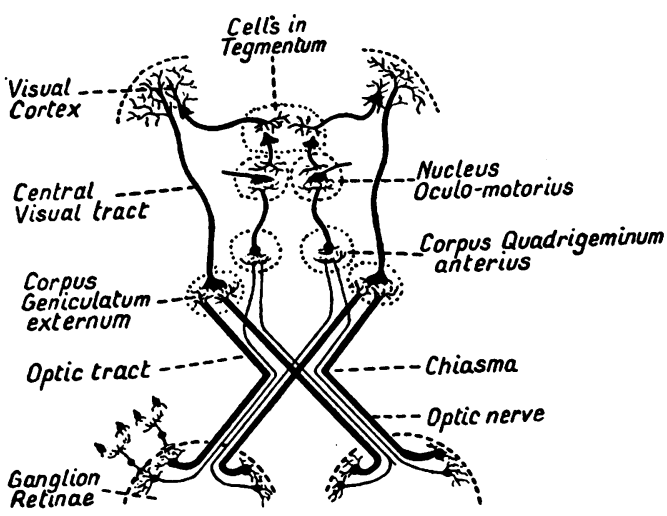

FIG. I.-Nervous Connexions of Visual Apparatus (After Schafer.)

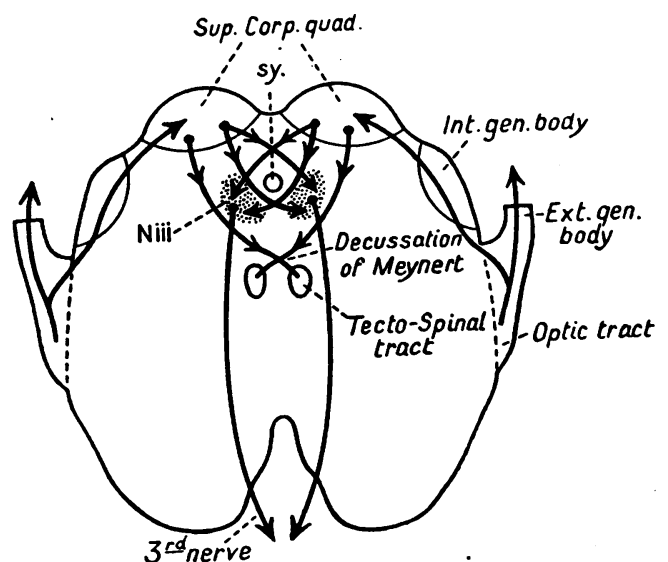

FIG. 2.-Section through Mid-Brain (diagrammatic). $\mathrm{N}$ iii = Nucleus of 3rd Nerve.

Sy. = Aqueductus Sylvil

The Argyll Robertson pupil in its typical form is small, reacts to accommodation convergence, but not to light, may occasionally dilate under light stimulation, and may be eccentric or irregular in outline. One pupil may be larger than the other. Non-syphilitic lesions of the same distribution may produce an Argyll Robertson pupil, as in encephalitis lethargica, disseminated sclerosis, tumour, syringo-myelia or syringo-bulbia, chronic alcoholism or trauma of the mid-brain. Traumatic lesions of the ciliary ganglion produce a total internal ophthalmoplegia. Encephalitis lethargica has been known to produce a reversed Argyll Robertson pupil that reacts to light but not to accommodation convergence. Reference must be made to the non-syphilitic Argyll Robertson pupil sometimes associated with absent tendon reflexes, the characteristics of which are as follows: There is no reaction of the pupil to light. During a sustained effort to converge there is a very 
slow contraction through a wide range, and slow dilatation with a definite latent period after the cessation of the act of convergence. This is called a myotonic reaction and is frequently unilateral; sometimes tendon reflexes are lost, and occasionally in cases which probably fall into the same group one pupil is found dilated and fixed with no reaction to light or convergence. The myotonic pupil was first described by C. Markus is I905. Foster Moore again drew attention to it in I93I, and in the same year Adie published a series of cases with absent tendon reflexes. The condition appears to be permanent when once established.

Tachycardia is an occasional finding in tabes and is of central origin.

Tabo-paresis :-When the signs and symptoms of both dementia paralytica and of tabes are present in the same case it is one of tabo-paresis.

Syphilitic Parkinson's Disease is a distinct, though rare, clinical entity, the lesion being striatal. In a case under the writer's care the patient is comparatively young and free from demonstrable vascular disease. The condition is resistant to anti-syphilitic treatment.

Syphilitic Vestibulo-Cerebellar Disorder:-Cases are occasionally encountered in which the presenting symptom is vertigo, no cause being found other than serological evidence of syphilis. The vertigo comes on suddenly in attacks and may cause the patient to fall. One such patient was a young adult male, who unfortunately died suddenly while awaiting admission to hospital for investigation, and no autopsy was obtained. Another, seen recently, developed frontal headache at the age of $5 \mathrm{I}$, eleven years after contracting syphilis. The headache was followed by vertigo on turning the head to the left. He was unsteady in walking with a tendency to fall to the left, and showed coarse nystagmus to the left and fine nystagmus to the right. The ears appeared normal and no defect of hearing was elicited. The blood Wassermann reaction was strongly positive, but the cerebro-spinal fluid was normal in all respects. All signs and symptoms cleared up rapidly on anti-syphilitic treatment. The exact site of the lesion cannot be stated in this case, but it seems likely that the vestibular nerve was involved, the cochlear nerve escaping. Sydney Scott has described a congenitally syphilitic child in which there was complete nerve deafness on one side with normal labyrinthine tests, and a dead labyrinth in the other side with normal hearing.

\section{Morbid Anatomy in Dementia Paralytica and Tabes.}

The macroscopic changes in the brains of general paralytics are as follows :-

1. Sometimes dural thickening.

2. Thickening and opacity of the pia-arachnoid which is adherent to the subjacent cortex, a worm-eaten appearance being left when the membranes are stripped from the brain.

3. Atrophy of the frontal and central convolutions of the brain, as the result of which there is a compensatory excess of cerebro-spinal fluid surrounding it and dilatation of the lateral ventricles.

4. The ependyma is thickened and roughened, especially in the fourth ventricle.

\section{Microscopic appearances:-}

1. In untreated cases the presence of trepanomata can be shown. 
2. A chronic lepto-meningitis consisting of a fibrosis and infiltration with lymphocytes and plasma cells.

3. Degeneration of cortical nerve cells with gliosis of the brain. It is this gliosis that produces adhesion between the brain and the meninges. fibres.

4. The presence of degenerating nerve fibres and loss of the tangential system of

5. Perivascular cuffing with lymphocytes and plasma cells.

In tabes, macroscopically there is thickening of the pia-arachnoid with some shrinkage in the size of the cord.

Microscopically, by appropriate staining, degeneration of the exogenous fibres in the posterior columns is shown, the condition being most marked in the lumbosacral region; also degeneration is found in the centripetal fibres in the posterior roots. The nerve cells in the posterior root ganglia may show degenerative changes, which are not, however, as a rule obvious.

\section{Congenital Neuro-Syphilis.}

Congenital neuro-syphilis may be present in the absence of other syphilitic stigmata. A suggestive family history is commonly, but not invariably, obtained when there have been multiple pregnancies.

It has been established beyond reasonable doubt by the work of Nabarro, in which the writer was privileged to participate, that syphilitic infection can be transmitted to the third generation through a congenitally syphilitic mother In these circumstances the infection seems prone to invade the nervous system.

The clinical manifestations of congenital neuro-syphilis are protean and include :-

1. Meningitis.

2. Ophthalmoplegia, internal or external.

3. Optic atrophy and choroido-retinitis.

4. Hydrocephalus.

5. Epilepsy, and infantile convulsions.

6. Syphilitic anterior polio-myelitis.

7. Nerve deafness.

8. Various cerebral lesions indistinguishable clinically from the primary degenerations.

(a) Cerebral diplegia (Little's Disease), in which are included hemiplegia, monoplegia, and quadriplegia.

(b) Parkinsonism, from involvement of the basal ganglia.

(c) Chorea and athetosis from lesions of the sub-thalamic region.

(d) General mental retardation with or without microcephaly from lesions of the frontal cortex.

(e) Pseudo-bulbar palsy.

9. Aberrations of mentality and conduct from milder degrees of involvement of the central nervous system, among which Nabarro includes an excessive nervous irritability, 
some mental retardation, undue naughtiness, and even downright delinquency. On the other hand, some of these children show a marked mental precocity.

10. Juvenile dementia paralytica, the usual age of onset being stated to be during the second decade; but it is frequently found earlier than this and has been observed as early as the age of five years. The mental change is one of progressive dementia, and delusions and euphoria do not occur. Epileptiform fits and emotional disturbances are common. The physical signs are the same as in the adult type of the diseasetremor, Argyll Robertson pupil, dysarthria, fits and increased knee jerks.

11. Juvenile tabes usually appears in early adolescence. Ataxia and lightning pains are rare. Pupillary changes, blindness, and loss of knee jerk are common.

12. Endocrine disorder cannot be excluded from a discussion of neuro-syphilis. The pituitary gland is likely to suffer in basal meningitis, and thus pituitary disorder is sometimes found in congenital syphilis, not necessarily accompanied by any other evidence of the infection. Any of the other endocrine glands may be affected; the gonads, the supra-renal, the thyroid, parathyroids, thymus and pancreas, and syphilitic lesions of these have been found post mortem. The usual result is some form of infantilism. Mongolism is not related to syphilitic infection.

As early diagnosis is essential in congenital neuro-syphilis if any benefit is to be hoped from treatment, serological examination of all children presenting signs and symptoms as described above should be a routine procedure.

\section{Changes in the Blood and Cerebro-Spinal Fluid.}

The serological tests for somatic syphilis: i.e. the Wassermann test and the various flocculation tests are all based on the same reaction between syphilitic antibody in the serum and a non-specific antigen. These tests are not, therefore, strictly specific. Variations in technique affect the sensitivity of the tests, and the results of different well-recognised tests on the same sera do not give completely harmonious results. However, there are few exceptions to the rule that a definitely positive reaction indicates syphilitic infection. A negative reaction, unfortunately, does not exclude with certainty its presence. Females during their reproductive period of life supply more false negative reactors than do males, or females after the menopause. In treated cases with neuro-recurrences, the blood Wassermann is negative in 40 per cent. (Merritt \& Fremont Smith).

The syphilitic antibody (a globulin), cannot pass through the choroid plexus or the normal capillary wall, but may do so when there is inflammation and congestion. Thus, in meningo-vascular syphilis with a positive reaction in the blood, a positive reaction in the cerebro-spinal fluid may be due to such passage and does not necessarily mean infection of the parenchyma of the nervous system. Other changes in the fluid that indicate a syphilitic meningitis consist of :-

1. A pleocytosis, mainly mono-nuclear.

2. An increase in protein.

3. The presence of globulin.

4. The property of precipitating colloidal suspensions, by virtue of the changed protein content. The precipitation of colloidal gold in this way is the basis of Lange's colloidal gold test. Other colloidal systems have been used in similar tests. 
The fluid, when withdrawn is usually clear and colourless and does not clot. In the rare instances where a gummatous meningitis has produced a sub-arachnoid block, the well known loculation syndrome of Froin is found with its high protein content and xanthochromia.

In dementia paralytica the Wassermann reaction in the blood is positive in 96 per cent. of cases, and in the cerebro-spinal fluid is positive in all. There is a moderate or slight pleocytosis, a protein increase according to the degree of meningeal reaction, and a "first zone" curve by the colloidal gold test (complete precipitation of the colloid in the first two to four tubes containing the highest concentrations of cerebro-spinal fluid-the so-called paretic curve).

In tabes the findings are variable. In early and untreated cases the changes cannot be differentiated from those found in dementia paralytica, except that the colloidal gold curve is more often of the "mid-zone" type (luetic curve). The Wassermann reaction is not invariably positive, but only in $70-80$ per cent. The Wassermann reaction in the blood is often negative (Stokes). Hazen (I928), states that the Blood Wassermann reaction is positive in 80 per cent. of tabetics, a figure, which, according to the impression of the writer, is too high especially where antisyphilitic treatment has been previously given. In cases that have become quiescent, the fluid may show no, or only very minor, changes. Despite this serological quiescence and the failure of the disease to advance clinically, lightning pains, crises and trophic effects may persist.

It is important to recognise that serological evidence of involvement of the central nervous system is present early in the disease in a considerable proportion of cases, usually in the form of a pleocytosis, though an increase of protein and a positive Wassermann reaction may also be found. These changes, present in about 30 per cent. of cases of early syphilis, frequently precede the advent of a secondary rash. The occurrence of a positive Wassermann reaction in the fluid before its appearance in the blood has been recorded by Wile \& Hasley (I92I), and by Mills (I929). It appears that invasion of the central nervous system, when it occurs, takes place within one to two years of the infection. In a large proportion the fluid returns to normal under ordinary treatment or spontaneously leaving a residuum of patients who are candidates for clinical neuro-syphilis in later years.

In congenital syphilis the cerebro-spinal fluid reactions are the same as in adults at the corresponding stage of infection. Thus, at the beginning of treatment the changes already described are often found in the absence of neurological signs, and frequently disappear under routine treatment, the changes persisting in a small proportion of cases. A blood Wassermann reaction that alternates between positive and negative after prolonged anti-syphilitic treatment particularly raises the suspicion of positive serological findings in the nervous system.

(The second part of this Article, "The prevention and treatment of neurosyphilis," will be published in our next issue). 